\title{
Kontrollierte Fragebogenentwicklung zur Messung erlebter Qualität von Produkten der Dräger Safety auf haptischer, optischer und akustischer Ebene
}

Julia Schneider, Christian Wölfel, Sarah Wandel, Michael Richenberger

Das Design eines Produktes gibt immer Hinweise auf seine Funktionalität, was es kann oder nicht kann, was es aushält oder nicht aushält. Die menschliche Wahrnehmung solcher Eigenschaften und Qualitäten kann daher wesentlich dafür sein, wie das Produkt gehandhabt und benutzt wird. Qualität wird durch formale Eigenschaften, Form und Detailgestaltung wahrgenommen. Diese muss mit der tatsächlichen Robustheit eines Produktes übereinstimmen, um den beabsichtigten Gebrauch und Einsatz des Produktes zu provozieren. Ein Missverhältnis zwischen erlebter und tatsächlicher Qualität eines Produkts kann zu Ausfällen, hohen Wartungskosten, geringerer Benutzerzufriedenheit oder geringerem Markenwert führen.

In der (interdisziplinären) kollaborativen Produktentwicklung basieren die Anforderungen und die Produktbewertung meist auf quantitativen Maßen. Während die Definition und Bewertung der tatsächlichen Qualität und Robustheit in der Produktentwicklung gut etabliert ist, fehlt noch eine standardisierte Methode zur Definition und Bewertung der erlebten Qualität eines Produkts.

In diesem Beitrag wird ein Ansatz vorgestellt, durch den die Übereinstimmung zwischen tatsächlicher und erlebter Qualität durch eine objektive Skala festgelegt bzw. kontrolliert werden kann. Dazu wurde eine semantisch-differentielle Skala entwickelt, die relevante Aspekte der haptischen, visuellen und akustischen Eigenschaften von Produkten erfasst. Es kann sowohl in der Anforderungsdefinition, als auch in den Evaluationsphasen und der Produktentwicklung des Designprozesses eingesetzt werden. Dadurch kann kontrolliert werden, ob die Designziele erreicht wurden oder nicht.

Keywords: Qualität, wahrgenommene Qualität, erlebte Qualität, User Experience, User Research, semantisches Differenzial 


\section{Motivation/Problemlage}

„Technik für das Leben“ - dafür steht Dräger. Aus der technischen Lösung Bier gleichmäßiger abfüllen zu können (dem Kohlensäure-Druckminderer: das Lubeca-Ventil) entstanden und über unzählige zukunftsweisende technische Lösungen gewachsen, sind wir bei Dräger oft sehr technisch getrieben. Wenn wir unsere Produkte betrachten, wollen wir jedoch möglichst alle Seiten betrachten. Auch die Zusammenhänge zwischen nicht-funktionalen Produkteigenschaften und dem Qualitätsurteil der Kunden. Welchen visuellen Eindruck macht ein Produkt im spannenden Moment der ersten Begegnung? Wie fühlt sich das Produkt in der Handhabung an? Und wie werden die Geräusche (auditiven Reize) des Produktes wahrgenommen? Dräger steht nicht nur für technische Lösungen, sondern auch für Qualität. Wie können wir also unsere Dräger Premium-Qualität systematisch erlebbar machen. Wir wollen mehr über die erlebte Qualität unserer Produkte erfahren und diese systematisch steuern können. Klassisches Qualitätsmanagement fokussiert auf tatsächliche technisch-funktionale Qualität.

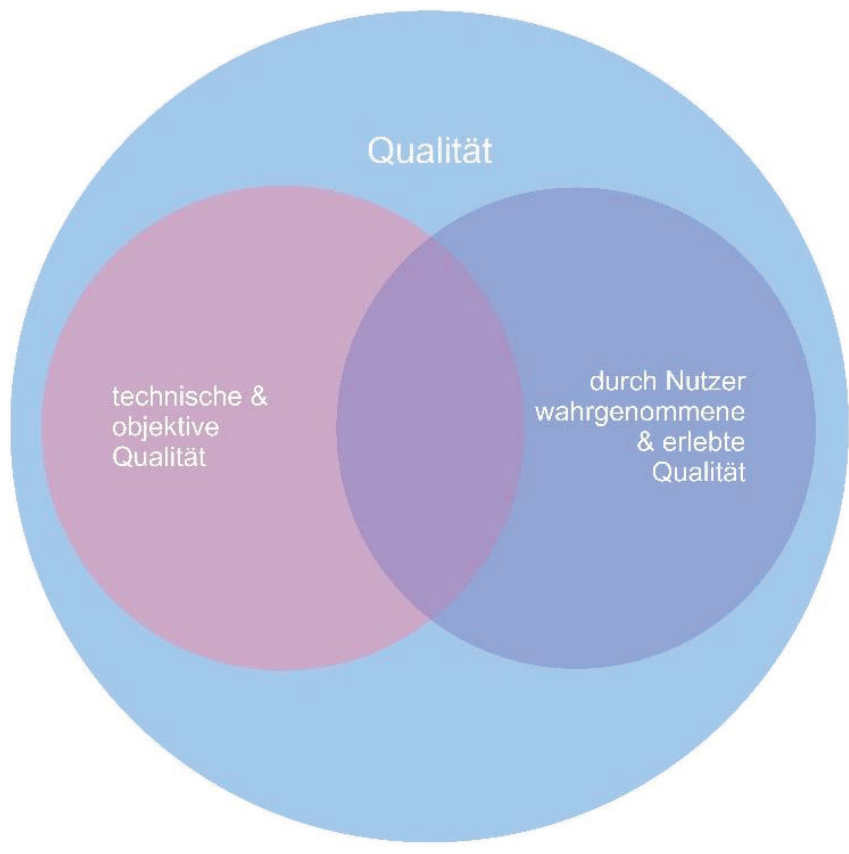

Abbildung 1: Zusammenspiel technischer und erlebter Qualität. 
Diese wird in vielen Unternehmen über rein technische Indikatoren, z.B. die Qualität oder der Preis verwendeter Materialien, oder die reibungslose Funktionalität von Sensorik und Technik bestimmt. Diese Aspekte sind und bleiben auch wichtig. Zusammenhänge zwischen anderen, auch nicht-funktionalen Produkteigenschaften und dem Qualitätsurteil der Kunden sind allerdings kaum bekannt und werden bislang kaum systematisch gesteuert. Wenn man als Entwickler allerdings erreichen möchte, dass ein Produkt als sehr hochwertig vom Nutzer wahrgenommen wird, ist es nötig sich die Nutzung zu diesen Produkteigenschaften genauer anzuschauen. Die objektive Technische Qualität eines Produktes ist nicht gleichzusetzen mit der erlebten Qualität des Nutzers. Diese zwei Ebenen des Konstruktes Qualität sollte in quantitativer und qualitativer Forschung an Produkten beachtet werden (Abbildung 1).

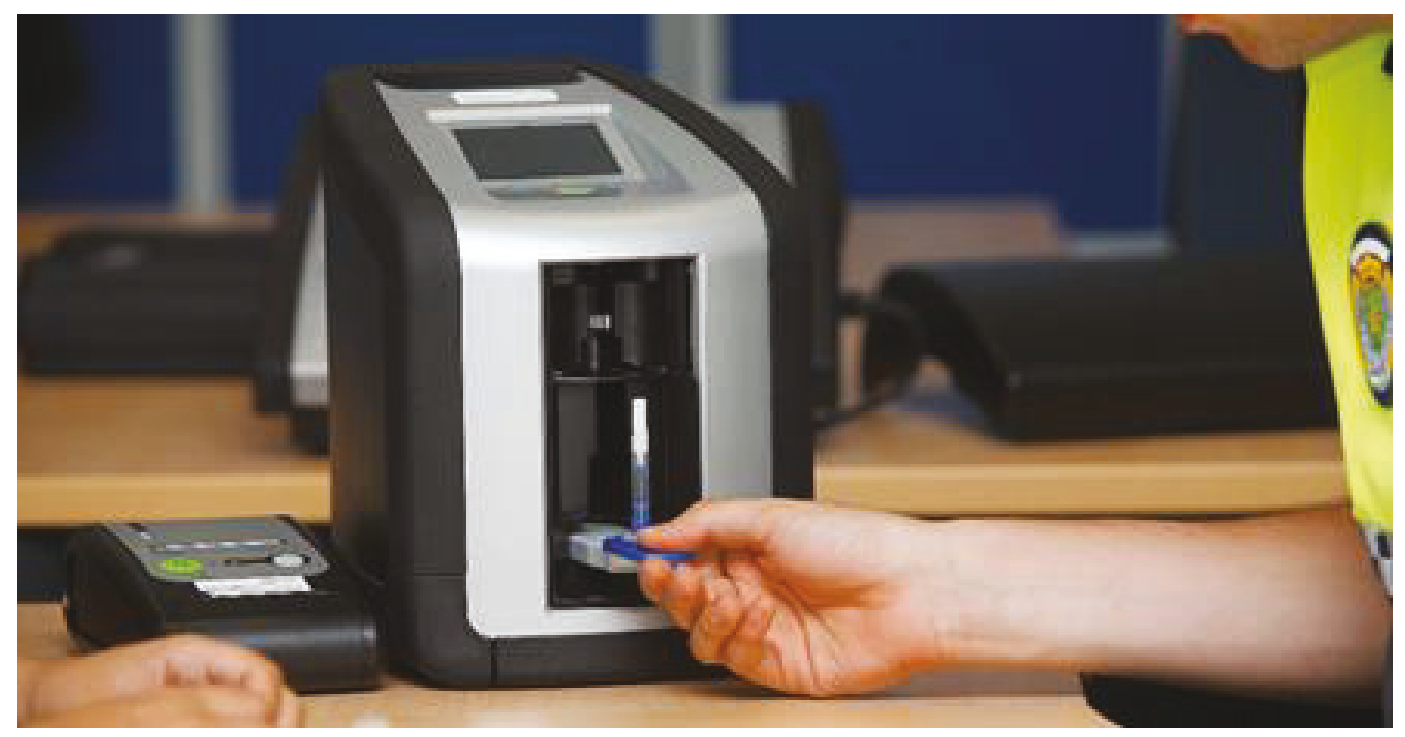

Abbildung 2: Dräger Drug Test.

Mit der TU Dresden haben wir ein Partner für dieses Ziel gefunden und konnten so im Rahmen eines Projektes (DrugTest NG, Abbildung 2) ein einheitliches Verständnis entwickeln, so dass die erlebte Qualität des Kunden eine Entsprechung in der tatsächlichen Produktqualität finden kann. Über verschiedene Stufen (Arbeitsschritte) wurden Fragebögen auf drei Subebenen erstellt, welche die visuelle, haptische und auditive Qualität eines Produktes abfragen und auf weitere Projekte übertragbar sind. Diese sollen in der angewandten Forschung dazu führen, dass die Dräger-Premium-Qualität für Kunden noch erlebbarer gemacht werden kann. 


\section{Theoretische Grundlagen}

\section{Erlebte Qualität}

Um die Dräger-Premium-Qualität messbar machen zu können ist es notwendig zu definieren, was eine erlebte Qualität überhaupt ausmacht und wie diese entsteht oder sich zusammensetzt. Im Cue-Modell (Olson \& Jacoby, 1972) wird im Bezug zu erlebte Qualität der Unterschied zwischen extrinsischen und intrinsischen cues (= Wahrnehmungselemente) für den Käufer beschrieben. Extrinsische Wahrnehmungselemente beschreiben dabei zum Beispiel Wertaspekte, Preis und Markenimage. Die intrinsischen wiederrum beziehen sich auf die Wahrnehmung des Produktes und wie dieses erlebt wird. Das schließt zum Beispiel physische Produktunterschiede mit ein. Im Allgemeinen haben alle dieser Wahrnehmungselemente einen Einfluss auf den potenziellen Käufer. Allerdings gibt es Evidenz dafür, dass einzelne Elemente einen stärkeren Effekt haben, als andere. Wie Szybillo und Jacoby (1974) in einer Studie zeigten, haben intrinsische Wahrnehmungsaspekte von Qualität etwa einen stärkeren Einfluss auf das Kaufverhalten, als die extrinsische.

Ein ähnlicher Ansatz zeigt sich auch in aktuellerer Forschung. So beschreiben Striegel und Zielinski (2018) zwei zentrale Aspekte für erlebte Qualität: Technische erlebte Qualität (TEQ) und wertbasierte erlebte Qualität (WEQ). Letzteres bezieht sich auf die generelle Erfahrung mit dem Produkt und kombiniert Produktcharakteristika mit externen Faktoren, wie zum Beispiel Kundenwerte, Markenwahrnehmung, Branding und vieles mehr. TEQ dagegen bezieht sich auf technische Produktaspekte und kognitive Merkmale des Produkterlebens. Die Autoren operationalisieren diese Aspekte auf der Sinnesebene des Menschen und definieren visuelle, taktile, auditive und olfaktorische Qualität. Die Operationalisierung über Sinnesebenen bietet eine nachvollziehbare und einfache Grundeinteilung für die Operationalisierbarkeit von erlebter Qualität. Diese Teilung wurde auch in den Arbeiten weiterer Autoren aufgegriffen. So beschreiben Stylidis, Wickman und Söderberg $(2015,2020)$ in dem von ihnen definierten „perceived Quality Framework“ (PQF) verschiedene Qualitätsattribute in einem komplexen Framework basierend auf den fünf Sinnesebenen visuell, taktil, auditive, olfaktorisch, gustatorisch mit drei Subebenen.

Die TEQ (Striegel und Zielinski, 2018), das PQF (Stylidis, Wickman, \& Söderberg, 2015, 2020) und die intrinsischen Wahrnehmungselemente (Olson \& Jacoby, 1972) bieten einige Parallelen. Dabei wird die Wahrnehmung eines Produktes und wie dieses erlebt wird in den einzelnen sensorischen Kanälen betrachtet. Alle Kanäle zusammen ergeben den letztendlichen Eindruck der Qualität beim Nutzer, wobei manche Ebenen je 
nach Produkt vorrangig sein können. Dieses Konstrukt die Grundlage für das hier vorgestellte Projekt (Abbildung 3). Bei vielen Unternehmen ist die extrinsische Ebene der Betrachtung noch immer wichtiger als die intrinsische Betrachtungsweise, und dass trotz der frühen Forschung zu diesen Themen. Um ein Produkt als hochwertig erleben zu können und einen Fokus auf den Premiumgedanken setzen zu können, ist die intrinsische Seite potenzieller Käufer extrem wichtig. Natürlich sollte diese extrinsische Ebene weiterhin betrachtet werden. Eine Ergänzung um die intrinsische Seite ist allerdings sinnvoll und in vielen Bereichen längst überfällig. Um diese Aspekte für Dräger mehr ins den Fokus rücken zu können, wurde mit der vorliegenden Untersuchung ein auf Dräger Wertvorstellungen angepasster Fragebogen entwickelt, der in der angewandten Forschung des Unternehmens zum Einsatz kommen wird. So soll eine Verbesserung der intrinsischen Qualitätswahrnehmung beim Kunden erreicht und messbar werden und somit auch langfristig stabil und noch besser werden.

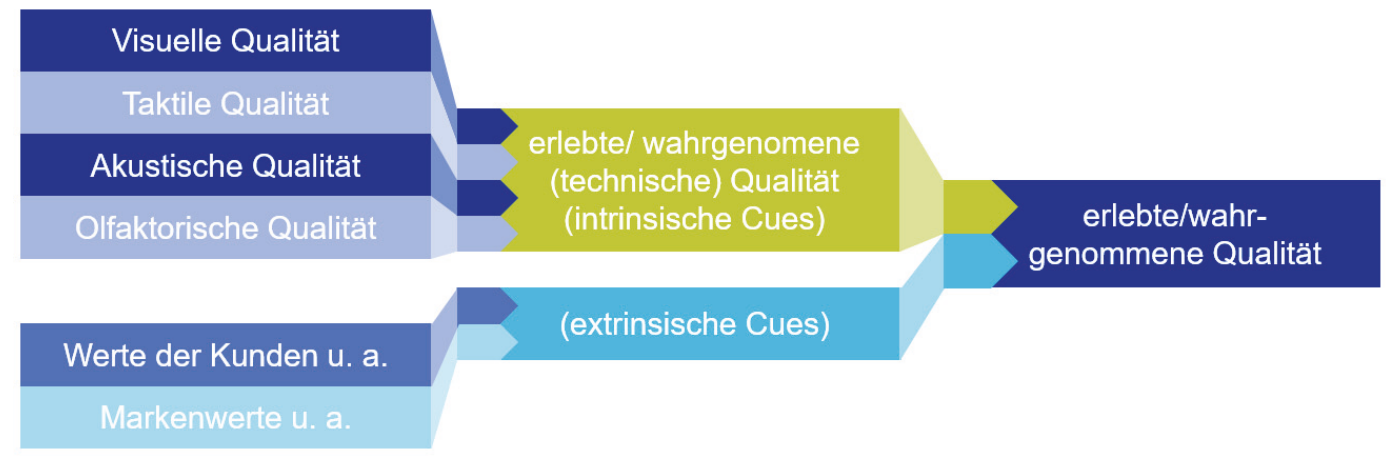

Abbildung 3: Grundlegendes Konstrukt der Arbeit, vereint Aspekte von Cue-Modell

(Olson \& Jacoby, 1972), TEQ und WEQ (Striegel und Zielinski, 2018)

und PQF (Stylidis, Wickman, \& Söderberg, 2015, 2020)

\section{Robustheit im Design}

Ein robust wirkendes Design impliziert, dass das Produkt äußeren Einflüssen standhalten kann. Ein gutes Beispiel für dieses Prinzip ist ein Baustellenradio (Abbildung 4). Es kann Stöße und sogar Stürze aus geringer Höhe aushalten, es kann Spritzwasser und Dämmerung aushalten. Es ist so konstruiert, dass es verschiedenen äußeren Einflüssen standhalten kann, die der Einsatzbereich mit sich bringt. Ähnliche Designkriterien wurden auch für Dräger Gasmessgeräte wie beispielsweise das Dräger Pac7000 (ein elektronisches Gasmessgerät, Abbildung 5) genutzt, um die Stabilität der Produkte für 
die Einsatzszenarien widerzuspiegeln. Im Gegensatz dazu, sind die Dräger Gasteströhrchen (Abbildung 6) aus Glas sehr filigran und schmal geformt. Ihre Form spiegelt die Funktionalität wieder, da sie zur Anwendung aufgebrochen werden müssen, können sie nicht besonders stabil sein. Die Fragilität führt aber gleichzeitig zu einer völlig anderen Handhabung, verglichen mit dem Pac7000, obwohl das Einsatzgebiet sehr ähnlich ist.

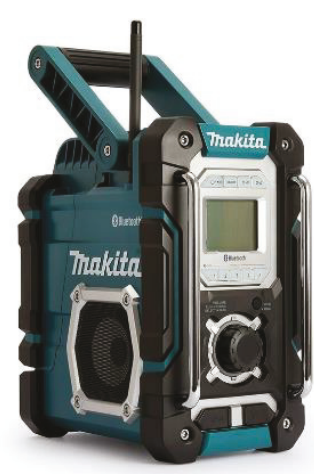

Abbildung 4: Baustellenradio

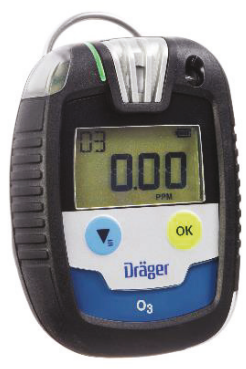

Abbildung 5: Dräger Pac7000

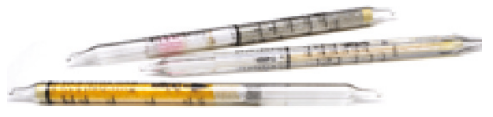

Abbildung 6: Gasteströhrchen

Unter diesen Gesichtspunkten kann das Design eines Produktes wesentlich für die Art der Handhabung durch die Benutzer sein. Ein Baustellenradio hat in der Regel dicke Kunststoffwände und ist umzogen von Gummikanten. Es sieht kräftig und stabil aus. Es spiegelt die Robustheit wider, die das Produkt in Wirklichkeit hat. Es sieht robust aus und es ist robust. Wenn die beiden Aspekte Design und Robustheit nicht zueinander passen, kann das zu Problemen führen. Ist ein Produkt sehr robust und stabil, aber sieht nicht so aus, kann es sein, dass das tatsächliche Nutzungsfeld von Aufgaben und Situationen, die es ausführen kann oder für die es gemacht ist, vom Benutzer nicht getestet werden und umgekehrt. Wenn ein Produkt wirklich stabil und robust aussieht, wird der Benutzer es wahrscheinlich auch so handhaben. Es wird möglicherweise mehr Stößen ausgesetzt, oder es wird in feuchten, nassen oder sehr Staubigen Umgebungen verwendet. In diesem Fall kann das Produkt durch die Handhabung Schäden erleiden, auch wenn diese meist unbeabsichtigt sind. In der Folge können hohe Kosten für ein Unternehmen entstehen, verbunden mit einer höheren Reklamations- und Reparaturquote. Darüber hinaus kann es zu einer geringeren Akzeptanz und Zufriedenheit des Anwenders mit dem Produkt und dem verweisenden Unternehmen führen. Die Anpassung des äußeren Erscheinungsbildes an die tatsächliche Robustheit 
des Produktes ist daher ein wesentlicher Punkt im Designprozess und kann bei Nichteinhaltung zu negativen Ergebnissen führen.

\section{Die Wirkung eines Produktes}

Semiotik ist die klassische Zeichenlehre. Konzepte der Semiotik finden im Kontext von Sprach- und Lerntheorien umfangreich Verwendung, sie können aber auch auf Produkte und ihre Gestaltung angewendet werden. Dabei bestimmen Geometrie, Farbe und Materialität die Semantik eines Produkts (Krzywinski \& Wölfel, 2020). Dieser semiotische Hintergrund spiegelt sich in der Eigenbedeutung des Produktes bezogen wider. So kann ein Produkt "hart" oder "weich", "aggressiv" oder "freundlich" wirken (Mühlbauer, 2021). Diese Aspekte sind ebenso relevant dafür, ob ein Produkt eher als robust oder filigran, als hoch oder minderwertig betrachtet wird. Allerdings ist das auch abhängig vom Produkt an sich, dem Kontext, der Erfahrung des Betrachtenden und weiteren Aspekten. Auch filigran aussehende Produkte können hochwertig vom Betrachter bewertet werden. Auch robust aussehend Produkte können als weniger hochwertig betrachtet werden. Wie bereits erwähnt ist für die angewandte Forschung die extrinsische Betrachtungsebene immer noch wichtiger als der intrinsische Ansatz. Um ein Produkt als qualitativ hochwertig erleben zu können, ist jedoch die intrinsische Seite extrem wichtig, wobei Mittel zur Messung der intrinsischen Qualitätswahrnehmung rar sind. Hassenzahl (2008) schlug den AttrakDiff-Fragebogen vor, der die Wahrnehmung der Attraktivität von Produkten misst. Das Tool basiert auf einem theoretischen Rahmen, der Grundlagen für verschiedene Aspekte der Produktqualität liefert, deckt aber nur Aspekte der visuellen Qualität ab; die anderen sensorischen Kanäle sind in seinem Ansatz nicht relevant. Im MeCue 2.0 (Minge, Thüring, Wagner, \& Kuhr, 2016) werden Produkte noch wesentlich ganzheitlicher abgefragt. So umfasst das Tool nicht nur Qualitäten und Attraktivität, sondern unter anderem auch Aspekte von Emotionen und Usability. Allerdings beschränken sich die Autoren auch hier auf die visuelle Ästhetik von Produkten. Aspekte anderer Sinneskanäle werden nicht mit betrachtet. Dies führt dazu, dass die meisten Autoren, die sich mit dem Thema der erlebten Qualität in einem oder allen möglichen Bereichen beschäftigen, ad hoc selbst erstellte Fragebögen zum Beispiel in den Bereichen Akustik und Haptik verwenden, die nicht auf einer validen Datenbasis beruhen (Yoshida, 1968; Mühlstedt, Jentsch, \& Bullinger, 2014; Kang \& Zhang, 2010; Raimbault, 2006; Wei \& Otto, 1992; Namba, et al., 1991; Lyon, 2003). Der Bedarf an einem universellen Instrument zur Messung der erlebten Produktqualität ist offensichtlich. 


\section{Methodik und Ergebnisse}

\section{Expertenworkshop}

Zur Generierung eines Wortpools für den geplanten Fragebogen wurde ein Workshop bei der Dräger Safety AG durchgeführt. Fokus wurde dabei auf verschiedene Ebenen gesetzt: Auf der einen Seite eine Hochwertigkeits-/Premiumdefinition für Dräger Safety Produkte, auf der anderen Seite die Generierung allgemeiner, mit Hoch- bzw. Minderwertigkeit assoziierter Begrifflichkeiten als Basis für ein Fragebogeninstrument. Um diese Aspekte von Qualität umfassend beschreiben zu können, wurden Referenzobjekte durch die Teilnehmer auf einer zehnstufigen Skala als hoch- bzw. weniger hochwertig eingeschätzt. Weiterhin wurden zu diesen Produkten passende Wörter generiert und gesammelt. Diese Daten boten die Grundlage für die Konstruktion des ersten Fragebogenentwurfes. In der Aufbereitung wurden semantische Differentiale für die Bewertung von erlebter Qualität für drei Sinnesebenen erstellt: Optik, Haptik und Akustik. Akustik wurde hier noch einmal in die zwei Subebenen natürliche und synthetische Töne untergliedert, da sich diese essenziell in der Tonbeschreibung unterscheiden.

\section{Datenerhebung und Itemreduktion.}

Die semantischen Differenziale aus dem Expertenworkshop wurden im nächsten Schritt anhand von Referenzobjekten (Akkus von Akkuschraubern) bzw. Tönen (Akkuschrauber Start und Stopp Töne, Dräger Drug Test Töne) auf einer siebenstufigen Skala bewertet. Es wurden Daten von N = 42 (N_männlich = 30; N_weiblich = 12; MW_Alter = 31,05; SD_Alter = 10,33) Probanden der TU Dresden und der Dräger AG erhoben und aufbereitet. Je Fragebogen wurde ein iterativer Prozess zur Itemreduktion und Generierung von Subskalen mit Hilfe von Varimax-rotierten Hauptkomponentenanalysen durchgeführt. Für den visuellen Teil wurden 18 Items auf vier Subskalen identifiziert. Die Varianzerklärung beträgt 71,17\%. Für den haptischen Fragebogen konnten 17 Items auf fünf Subskalen identifiziert werden. Die Varianzerklärung beträgt 68,03 \%. Für natürliche Töne wurden 19 Items auf 5 Subskalen identifiziert. Die Varianzerklärung beträgt 72,47 \%. Für synthetische Töne wiederum, wurden 18 Items auf fünf Subskalen gefunden. Die Varianzerklärung beträgt 71,41\%.

\section{Diskussion \& Implementation bei Dräger}

Die grundlegende Perspektive der vorliegenden Untersuchung war die Entwicklung eines Fragebogens zur Messung hoher Qualität als Indikator für Robustheit. Basierend auf dem PQF (Stylidis, Wickman, \& Söderberg, 2015, 2020) wurde die theoretische 
Struktur des Fragebogens in die verschiedenen Sinneskanäle unterteilt. Ein Expertenworkshop zu Generierung eines semantischen Differentials, sowie eine Datenerhebung wurden durchgeführt, um eine empirische Basis für einen Item-Reduktionsprozess zu schaffen. Das Ergebnis sind vier verschiedene Fragebögen, jeweils einer für haptische, visuelle, natürlich-auditive und synthetisch-auditive Signale. Die Module der Fragebögen wurden separat entwickelt, getestet und können daher je nach Einsatzziel flexibel und einzeln oder zusammen eingesetzt werden. Da die Anzahl der Items pro Fragebogen gering ist (minimal 17, maximal 19), ist das Tool relativ einfach zu bedienen und ein flexibler Einsatz für jede Art von Analysen in einem komfortablen Zeitrahmen möglich. Die Originalsprache ist Deutsch; eine Validierung für das Tool in englischer Sprache liegt noch nicht vor.

\section{Einschränkungen}

\section{Generalisierbarkeit}

Die Fragebögen zur erlebten Qualität können für kleine und handgehaltene Produkte verwendet werden, da die Datenbasis für diese Produktgruppe erhoben worden ist. Für Software-Schnittstellen ist der Einsatz nicht zu empfehlen. Für den Einsatz bei große industriellen Technologien oder Webseiten kann keine valide Anwendbarkeit angenommen werden. Studien müssen zeigen, ob die Skalen für diese und andere Anwendungsfälle adaptierbar sind. Für die auditive Skala ist eine Anwendbarkeit für Website- oder Interface-Aspekte denkbar, dazu sind allerdings weitere Untersuchungen notwendig. Weiterhin ist eine Globalbewertung der erlebten Qualität mit der aktuellen Version des Fragebogens nicht vorhanden.

\section{Olfaktorische und Gustatorische Qualitäten}

Zum PQF (Stylidis, Wickman, \& Söderberg, 2015, 2020) gehören nebst der betrachteten drei Sinnesebenen (visuell, auditiv, haptisch) noch die gustatorische und die olfaktorische Ebene. Für das aktuelle Projekt wurden diese Aspekte bewusst außer Acht gelassen. Dies lässt sich durch den explorativen Charakter der Fragebogenerstellung begründen und mit der Tatsache, dass Geruch und Geschmack als für die primär betrachteten Produkte der zu testenden Produkte zunächst untergeordnet zu betrachten sind. Dennoch sollte die Wichtigkeit dieser Sinneskanäle für mögliche andere Einsatzszenarien nicht unbeachtet bleiben. Es ist immer wichtig im Vornherein zu bedenken, welche der Ebenen relevant für ein zu testendes Produkt sind. Insbesondere die olfaktorische 
Ebene sollte hier in Zukunft mit eingebunden werden, da sich aus den Untersuchungen gezeigt hat, dass einige der Probanden eine Qualitätsbewertung auch über einen beispielsweise unangenehmen Geruch der Testprodukte erschließen.

\section{Stichprobengröße}

Die Stichprobe von N = 42 kann ambivalent betrachtet werden. Aus Standardliteratur lassen sich häufig Konventionen für eine Stichprobengröße von N > 200 Probanden finden. Insgesamt sind Meinungen zur Stichprobenproblematik allerdings ambivalent (Field, 2019). MacCallum, Widaman, Zhang und Hong (1999) fanden heraus, dass auch eine Faktorenanalyse, bei der alle communalitäen >. 6 sind, auch bei Stichproben < 100 stabile Ergebnisse liefert. In der aktuellen Studie sind alle diese Kommunalitäten durch den iterativen Prozess >.6.

Die so genannte KMO Statistik (Kaiser-Meyer-Olkin Kriterium) ist ein Maß der Angemessenheit einer Stichprobe. Nach den Konventionen von Kaiser und Rice (1974), sollten die Werte nicht unter .5 fallen. Höhere Werte indizieren reliable und verlässliche Faktoren. Für die Ergebnisse der aktuellen Untersuchung liegen die KMO Statistiken zwischen .58 und .75 also in einem akzeptablen bis mittelguten Bereich.

Weiterhin wurde für die Datenerhebung wurde ein multifaktorielles Design mit mehreren Referenzprodukten erhoben, die alle komplett bewertet wurden. So ergibt sich aufgrund mehrerer Fälle pro Proband eine größere Datenbasis als zunächst offensichtlich (Fallanzahl zwischen 84 und 126). In jedem Fall ließen sich in der statistischen Auswertung gute Werte zwischen $68.03 \%$ und $72.47 \%$ für die Varianzerklärung feststellen.

\section{Ausblick}

In den nächsten Schritten sollen die entworfenen Fragebögen praktisch für den Einsatz in der Produktentwicklung getestet werden. Für den aktuellen Stand der Forschung wird vorgeschlagen, sie nur für kleinere Geräte zu verwenden, da die Datenbasis für Handheld-Objekte erhoben wurde. Für den Einsatz der Fragebögen für große industrielle Technologien kann keine valide Anwendbarkeit angenommen werden. Ob die Skalen für diese und andere Anwendungsfälle adaptierbar sind, muss erst in Studien geprüft werden.

Der Fragebogen erhebt Maße für Aussehen, Gefühl mit der Materialität und Tonalität. Diese Dinge beschreiben zu können ist ein erster wichtiger Schritt in die Richtung „Premium erlebbar machen“. Allerdings ist es so, dass semiotisch gesehen nicht jedes der semantischen differentiale entweder als hoch- oder als minderwertig empfunden wird. 
Es gibt durchaus auch Items im Fragebogen, die sowohl das eine, als auch das andere beschreiben können, ein Beispiel wäre "matt" oder "glänzend“. Beides kann hohe Qualität ausstrahlen. In nächsten Schritten zum Fragebogen, sollen diese Ebenen noch weiter betrachtet werden, sodass ein globales Maß der der verschiedenen Qualitätsmaße ermöglicht wird.

Die Fragebögen wurden bei Dräger bisher bereits in mehreren Projekten angewandt, hierbei aber nur der haptische und der visuelle Teil. Ein konkretes Beispiel betrifft den Drug Test, der zu Beginn dieses Betrages bereits angesprochen wurde (Abbildung 2). In diesem Projekt wurden für die nächste Generation zwei verschiedene Design Prototypen auf der visuellen Ebene verglichen und um qualitative Fragestellungen erweitert. Ein weiteres Projekt ist das „new Softpack", bei verschiedenen Prototypen einer neuen Tasche einer Fluchthaube miteinander verglichen wurden. Auch hier wurde der Fokus auf die visuelle Ebene gelegt. Dem Projektteam war besonders wichtig, dass die neue Tasche sehr robust auf die Probanden wirkt. Auch hier wurde der visuelle Fragebogenteil um qualitatives Feedback erweitert. Somit konnten Gründe für auseinandergehende Urteile auf der Skala direkt erfragt werden

In Zukunft wird es spannend sein, auch mehr über das auditive Feedback zu erfahren und die Fragenbögen vermehrt in quantitativen Umfragen einzusetzen. Mit den praktischen Erkenntnissen sollen weitere Schritte der Fragebogenvalidierung geplant werden.

\section{Literatur}

Zielinski, D., \& Striegel, S. S. (2018). Perceived Quality of the Split-Line Design and Quality. Proceedings of the DESIGN 2018 15th International Design Conference, (S. 453-464).

Field, A. (2019). Discovering statistics using IBM SPSS statistics. Sage.

Hassenzahl, M. (2008). User experience (UX) towards an experiential perspective on product quality. Proceedings of the 20th Conference on I'Interaction Homme-Machine, (S. 11-15).

Kaiser, H. F., \& Rice, J. (1974). Little jiffy, mark IV. Educational and psychological measurement, 34(1), S. 111-117.

Kang, J., \& Zhang, M. (2010). Semantic differential analysis of the soundscape in urban open public spaces. Building and environment,. 45(1), S. 150-157.

Krzywinski, J., \& Wölfel, C. (2020). Industriedesign und nutzerzentrierte Produktentwicklung. In B. Bender, \& K. Gericke, Pahl/Beitz Konstruktionslehre (S. 684-704). Berlin: Springer.

Lyon, R. (2003). Product sound quality-from perception to design. Sound and vibration, 37(3), S. 18-23.

MacCallum, R., Widaman, K. F., Zhang, S., \& Hong, S. (1999). Sample size in factor analysis. Psychological methods, 4(1), S. 84.

Kontrollierte Fragebogenentwicklung zur Messung erlebter Qualität von Produkten der Dräger Safety auf haptischer, optischer und akustischer Ebene 
Minge, M., Thüring, M., Wagner, I., \& Kuhr, C. V. (2016). The meCUE questionnaire: a modular tool for measuring user experience. In Advances in Ergonomics Modeling, Usability \& Special Populations (S. 115-128). Cham: Springer.

Mühlbauer, F. (2021). Der Einfluss stilistischer Merkmale einer Entwurfsvisualisierung auf die semantischen Produkteigenschaften (Dissertation, Design). Dresden: Technische Universität Dresden.

Mühlstedt, J., Jentsch, M., \& Bullinger, A. C. (2014). Haptische Textur vs. optische Struktur-Haptik im Spannungsfeld von Leichtbau und Stabilität. (G. eV, Hrsg.) Gestaltung der Arbeitswelt der Zukunft, S. 60.

Namba, S., Kuwano, S., Hashimoto, T., Berlund, B., Da Rui, Z., Schick, A., ... Florentine, M. (1991). Verbal expression of emotional impression of sound. Journal of the Acoustical Society of Japan, 12(1), S. 19-29.

Olson, J. C., \& Jacoby, J. (1972). Cue utilization in the quality perception process. ACR Special Volume.

Raimbault, M. (2006). Qualitative judgements of urban soundscapes: Questionning questionnaires and semantic scales. Acta acustica united with acustica, 92(6), S. 929-937.

Striegel, S. S., \& Zielinski, D. (2018). Perceived quality of the split-line design and quality. DS 92: Proceedings of the DESIGN 2018 15th International Design Conference , (S. 453-464).

Stylidis, K., Wickman, C., \& Söderberg, R. (2020). Perceived quality of products: a framework and attributes ranking method. Journal of Engineering Design, 31(1), S. 37-67.

Stylidis, K., Wickmann, C., \& Söderberg, R. (2015). Defining perceived quality in the automotive industry: An engineering approach. Procedia CIRP, 36, S. 165-170.

Szybillo, G. J., \& Jacoby, J. (1974). Intrinsic versus extrinsic cues as determinants of perceived product quality. Journal of Applied Psychology, 59(1), S. 74.

Wei, K. C., \& Otto, N. C. (1992). Engine sound quality evaluation using semantic differential method, CA: SAGE Publi. Proceedings of the Human Factors and Ergonomics Society Annual Meeting . 36, S. 1244-1248. CA: Los Angeles: Sage Publications.

Yoshida, M. (1968). Dimensions of tactual impressions. Japanese Psychological Research, 10(3), S. 123-137.

\section{Kontakt}

Sarah Wandel, M. Sc.

Dipl.-Des. Michael Richenberger

Dräger Safety AG \& Co. KGaA

Revalstraße 1

23560 Lübeck, Germany

www.draeger.com
Julia Schneider, M. Sc.

Dr.-Ing. Christian Wölfel

Technische Universität Dresden

Professur für Technisches Design

01062 Dresden

www.tu-dresden.de/design 